\title{
New Vistas in Drug Design Analysis: Computational Approach to Pharmacokinetics and Pharmacodynamics Tool Development
}

\author{
Deepalakshmi R \\ Research Scholar, \\ Dept. of Computer science, \\ Presidency College, \\ Chennai-5
}

\author{
Jothi Venkateswaran C, \\ Ph.D \\ Research Supervisor, \\ Associate Professor \& Head, \\ Dept. of Computer science, \\ Presidency College, Chennai-5
}

\author{
Priya Shankar \\ Managing Director \& \\ Cofounder, \\ Xinnovem Technologies, \\ Chennai
}

\begin{abstract}
The Pharmacokinetic/Pharmacodynamic (PK/PD) modeling associated simulation could as well be used as an 'applied science' tool to seek effective end results. The latest medication ensuring and enhancing safety, affordable cost and quicker results are greatly anticipated. The PK/PD modeling might be used from the run through all clinical phases of drug development. The best use of PK/PD modeling and simulation sometimes may result with unsuccessful compounds and study failures and at times a few studies might require registration. In order to demonstrate the potentiality of the PK/PD modeling in drug development, it has to be embraced by the business men and the restrictive agencies, and in addition to it, people have to be educated upon the subject. The Mechanism-based Pharmacokinetic-Pharmacodynamic (PK/PD) modeling is a popular computational method used for simulating the drug treatment of infectious diseases. It possesses the potential to reinforce our understanding of drug treatment outcomes, drug readying methods, and dosing regimens. A computational developed tool though expensive can address issues such as weak patient compliance and drug resisting power. The methodologies used in the previous PK/PD models limits the user approach as it needs computer literacy to operate the tool. An attempt is made to create a user friendly tool, the PK/PD which could be operated at ease by anyone and everyone.
\end{abstract}

\section{General Terms}

Computational developmental tool, PK/PD.

\section{Keywords}

Pharmacokinetics, Pharmacodynamics, drug treatment, software tool

\section{Availability}

The live demo of this software is provided in the link below: www.xinnovem.in/pkpd.html

\section{INTRODUCTION}

The computer buoyed Pharmacokinetic / Pharmacodynamic approach is applied to quantify the disposition of pharmacologic effects of the drug, explore the exposureresponse relationships, and predict the safety and effective outcomes [1]. The exact design of the drug and the risk of applying environment toxins absolutely depend on thorough knowledge of PK/PD. The frequently used methodology to scale up the usage of PK/PD stretches out upon physiologically based pharmacokinetic modeling (PBPK) [2].
The process of drug development could be a complicated and more expensive one. In the bygone days, the transportation to replace a drug for promotion was anticipated to be between $\$ 800$ million and $\$ 1$ billion. Currently, these candidate drug compounds are under testing. Less than $10 \%$ of medicine in the clinical trial is boosted up to the approval section. Two key reasons attributed to the failure of medicine at the later stages are firstly the lack of understanding of the link between the dose-concentration response and out of the blue safety events. Given to this state of affairs, it is necessitated to develop a tool which facilitates the prediction behavior of a drug that can perform in vivo and assist within the success of a clinical therapeutic

The Pharmacokinetics (PK) characterizes the ADME characteristics of the drug specifically Absorption, Distribution, Metabolism, and Elimination properties of a drug [3]. The Pharmacodynamics (PD) defines the physiological and biological response of the administered drug. The PK/PD modeling establishes the associations between the mathematical and theoretical connections and as well assists in the higher prediction of the drug action. The Integrated PK/PD modeling enjoys a greater significance in gauging the several drug development programs [4].

PK/PD testing is often performed at each stage of the drug development method. As a result of development, it turns into more and more complicated, time taking, and price intensive process. Drugs manufacturing firms demand to form higher use of PK/PD knowledge to eliminate weaker candidates at the start and determine those with the most effective probability of clinical success.

\section{STREAMLINING THE METHOD}

A major issue in the drug development method is time. Prognostic modeling tools will give priceless data to raise the drug development method. Pharmaceutical corporations historically perform serial testing of drug candidates by screening and choosing the most effective performers at each section of the clinical drug-development cycle. This may take as long as six to ten years and price many hundred million dollars. Hence, it's imperative for workplaces to adopt technologies that improve the standard of the drug development method and improve speed to plug.

"PK/PD modeling will greatly compresses timelines by sanctioning offices to utilize PK/PD information collected in clinical test trials," says Graham. "Instead of a serial approach, modeling permits a parallel approach, serving to 
jump-start clinical test and phase III trial styles. This may considerably cut back the event time at each section. Additional advantages of PK/PD modeling are optimized dose regimens leading to smarter clinical test and phase III trials with a minimized risk of drug failure at the main stage." Fig. 1 depicts the streamline development of PK/PD process.

Traditional PK/PD modeling in drug development defines parameters like drug dose concentration, drug exposure effects, drug half-life, and many drug impacts relative to current trends. "When used at the ground line, techniques like drug, disease, trial and market modeling will enhance the whole development method, which ends in higher choices through express thought of risk and higher utilization of data," presented by Mark Hovde, senior vice chairman, Pharsight opposition., Mountain read and Calif.

It is also said that, implementation of PK/PD modeling approach may be a difficult task. "One needs to spend time and resources up front within the drug development method [5]. From a modeling stand, one must establish and build into the trial style frequent measures of clinical effectively and/or toxicity similarly as representative biomarkers for worth modeling," depicts Graham.

\section{DETERMINING RELATIONSHIP}

PK/PD modeling approaches have proven samples of analyzing relationships among the biomarker responses, drug levels, and dosing rules. The PK/PD details of a drug volunteer and the capability to predict a patient's response are important to the triumph of clinical trials. The recent advances in the biological techniques and a stronger understanding of targets for numerous diseases have valid biomarkers as an honest clinical indicator of a drug's therapeutic effectiveness.

"Biomarker facilitates in establishing a biological response to a drug candidate. Once a biomarker is clinically valid, the trial simulations are often effectively modeled. The Biomarkers have the potential to realize the surrogate condition that will sometime substitute for the clinical outcomes in the drug development," depicts Peter, the Principal at MDS company Services, Montreal, Canada. Some current technologies are used to develop the biomarker at intervals of the realm of ligand binding and liquid chromatography/tandem mass spectrographic analysis [6]

\section{EXISTING TOOLS FOR PK/PD}

A numerous, varied PK/PD modeling tools are handy in the market to facilitate the drug development researchers, and one amongst them is Pharsight's WinNonlin. Hovde asserts "With shored up knowledge such as the presence of drug in plasma at specific points in time, and permitting for calculation and estimation of essential PK parameters in the vein of concentration, total exposure (i.e., space below the curve), half-life, clearance rate, and volume of distribution, PK/PD modeling accomplishes desired outcomes".

WinNonlin reciprocally supports customary as well as userdefined models to deal with any reasonable knowledge. Its worksheet interface smoothes the progress of knowledge transformations, and it as well offers modeling engines that puts forward versatile pre and post-modeling analyses. The additional tools modify exploration of a drug's properties in the course of the statistical approaches.

In case of the available data for analysis [7] is skimpy during the clinical trials of phase III PK/PD modeling is most handy. It speaks of the differences among the individuals involving both healthy and diseased patients. Formerly, the testing was carried out only upon a small homogenous group as a group of rats, or the same age of people, etc.; however this tool is executed upon a diversified heterogeneous population. The later is recommended for the drug tested upon a target population drives to larger avenues.

Robert J. Baner flung out an appropriate PK/PD software package to back up the population analysis. An overview of the software that exposes the present population analysis methods and the software packages benefitting to resolve various PK/PD modeling problems is provided. Four different algorithms NONMEM, Win BUGS, MONOLIX and SADAPT are considered for analysis. NONMEM (Non-linear Mixed Effect Modeling) is discerned to be the best and accurate compared to other models [8]. NONMEM is also explicitly used compared to other packages. [9- 13]

Statistical techniques gained promise in the comparative study of population methods [14-16]. The frequently used methods in the population analysis are semi-parametric, nonparametric and Gaussian-Quadrature. The software and the statistical comparisons of the population methods namely naive-pooled data analysis, 2-stage, global 2-stage, linearized expectationmaximization (EM) (iterative 2-stage), and nonlinear mixed effects are methods using first-order and Gaussian-Quadrature methods, nonparametric, and semi-parametric methods have been explored in earlier reviews [5-7]

Kinetica a rebuilt model is furthermore a new tool that is ready-to-use PK/PD model. It eases a quick analysis and reporting of data. Nevertheless, it is awfully expensive. In addition, the results obtained thereof are not accurate.

PKSolver is Microsoft Excel software written in visual Basic and intended mainly to solve PK/PD. Multiple Absorption site (MAS) and Enterohepatic circulation are the two built-in models used here. The Output report is displayed in the MS Word. However, this newly created tool cannot clearly plot the graphs in the software [14].

The other idea from Pharsight is that the Knowledgebase Server (PKS). This platform abets the technical infrastructure to manage PK/PD knowledge and the associated analyses through the development programs. The information gathered is captured, stored, managed, analyzed, and reformed into a secure, regulatory-compliant format. "PKS permits the automation of $\mathrm{PK} / \mathrm{PD}$ progress and may be an amazing time saver," Hovde says.

Delays throughout clinical development as well add up to expenses and leads to the greater loss of revenues. Pharsight's Trial machine software package authorizes the clinical development team to check hypothetic eventualities and simulates trials to assess the probability range of trial outcomes. Trial machine software package permits researchers to mix PK/PD modeling knowledge with the population PK/PD knowledge. The impact of every variable, inclusion/exclusion criteria, and dropout rates can even be studied to style an optimum trial. The Trial simulations are often simply updated as the new relevant clinical knowledge befalls in the market.

"With a lot of data drawn from a given subject population, the added advantage of the trials are less significant, more rapid to enroll, and of squat value," says Hovde [15]. "To investigate the early signals of drug impact, some firms are risking terribly high-replicate trial simulations in the earlier stages of development [16]. 


\section{PHASES OF CLINICAL TRIAL}

1. Lab study The cells will be left to react with the drug. The value of PK is found.

2. Animal study Drug is consumed by the animals. The reactions are observed and noted.

3. Drug is masticated by Human and volunteers of (620) too. The reactions are studied.

\subsection{Clinical trials}

Phase I- 50 persons

Phase II - 100 persons

Phase III - 1000 persons

The drug at each phase is sent to the Food \&Administration (FDA) section. At the completion of verification at the Phase III, it is sent for FDA approval. Once the process of authentication is fulfilled, the drug is sent to the market for sale. A lifetime approval is ensured. The Drug is gulped down for 5 days and it remains in the body for 90 days. Sooner or later, they consume the next drug. In order to save time, money and people, the computer operators along with bioinformaticians have developed many types of software. This opens possibilities for the effective development of PK/PD tool.

\section{METHODS}

The model created is a user-friendly application embossed with Graphical User Interface (GUI) that integrates parameters and methods together.

\subsection{Softwares Used}

\section{Front end: $\quad$ Microsoft Visual studio 2005}

Back end: $\quad$ Microsoft SQL Server 2008

The front end tool Visual Basic is chosen as it is more userfriendly and supports all mathematical calculations to be done in a simpler method. SQL server 2008 is the latest version which proves to be best in all database accession and retrieval methods, coding's used in these are even more user friendly. Advantages of using VB and SQL over other software's are as follows.

(i)Error handling is made easy.

Underlying PK/PD models generally consists of systems of ordinary differential equations, were written using java script, graph was constructed with. Project file are given as exe file which can be run by any user provided these two software must be installed in their system which in turn can be freely

Reference Test $=\mathrm{t}($ Ref $)-\mathrm{t}($ Test $)$

Tmid $=($ Current time + Previous time $) / 2$

$\Delta \mathrm{M}=$ Current batch Ref time - Previous batch Ref tim

$\mathrm{T} * \Delta \mathrm{M}=\quad(2) *(3)$

Similarity factor $=50 * \log \left(\left((1+(1 / 9) * \text { sum of values of Ref test })^{\wedge}\right.\right.$. $\left..5)^{*} 100\right)$

$\mathrm{F} 1=($ Sum of Ref test/Dissolution value of ref batch at time $)(6)$

$$
\text { Difference factor }=\sum \frac{(\text { ref test }) \quad * 10}{\left.\sum \text { (batch ref test }\right)}
$$

downloaded from web. Working of tool fully is given as a eleven minutes video demo in tool.avi, which can be downloaded and viewed. Reason for choosing Visual Basic is as given below:
- The format of the language is easy, and it is the best integrated language used for Interactive Development Environment ("IDE").

- The graphical user interface (GUI) gives a classic view for running of the program. Its online help system makes the application run even easier.

Reason for choosing SQL 2008 is easier Encryption, External key management, Auditing, Data synchronizing and spatial data management.

\subsection{Superiority of the model or software}

At the moment only a few such as Math work software's are available in the market. The performance of the tool it should be in MAT format. This software is in command prompt, which is not user friendly [17]. As far as MATH work software's are concerned, no doubt, many online tools are available which is costlier and it can be used or operated only by a computer savvy.

Most of the tools available are difficult to use by the persons who have meager knowledge in computer [18-23]. To fill the gap of the above tools we developed a software PKPD kit.

The PK details are entered by the user at three phases such as fast, medium and slow. The similarities of all the three factors are perceived. The Difference factor F1 is also calculated. Based on the table and the mathematical formulae given below, both the similarities and F1 are calculated.

\section{RESULTS}

In PK/PD tool the screens are organized into project.

Begin the experiment with relevant parameters

The comparison of dissolution profile can be achieved using difference factor (f1) and a similarity factor (f2) which emerges from simple model approach. Difference factor f1 is calculated as follows:

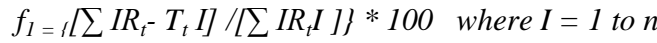

Here, $\mathrm{n}$ denotes time points. Rt denotes the dissolution value of the reference batch at time $t$ and $\mathrm{Tt}$ is the dissolution value of the test batch at time $t$.

Similarity factor is calculated based on the following formula

Similarity factor $f 2=50 * \log (((1+(1 / n) *$ sum of values of Ref test)^-.5)*100) [24].

The Similarity factor is a logarithmic reciprocal square root transformation of the sum squared error and is a measurement of the similarity in the percent dissolution between the two curves.

The Mean Dissolution T time (MDT) is the mean time for the drug to dissolve under invitro dissolution conditions. This is calculated using the equation

$M D T=\int_{0}^{\infty}\left(M_{\infty}-M(t)\right) d t / M_{\infty}$

List of Mathematical formulas used in our software development are depicted from Equations numbered (1) to (7).

Using the formulas above the values of Table 1 as depicted below are calculated for Dissolution profile of Drug x in Fast, Intermediate and Slow medium respectively.

Run the experiment with SQL Server as the backend to store and retrieve the records.

View the result as a graph. 
Save and explore the result. Results will be saved in database. The snapshots shown in Fig 2 demonstrate the execution of the tool. The values are entered for the fast, the slow and intermediate dissolution of the drug. The first screen starts from the user login and registration. The PK dissolution screens for the fast, the medium and the slow are categorized. The graph furnishes the output and the convergence of all the three modes. The graph in Fig. 2, shows all the three values of Fast, Intermediate and Slow converges and hence the above drug can be preceded for next step.

\section{DISCUSSION}

The extended PK/PD mechanism based modeling is applied to the drug dosage type and the new developed tool is very much user friendly. The login screen checks with the database whether the user has correctly logged in, and if he/she is a new user, and immediately asks for registration. After the registration, the drug type and the drug name along with the time interval taken for the drug to be reacted are entered by the user. The PK dissolution factor for the fast, the intermediate and the slow is entered. Using the mathematical formulae as said above, the values are typed manually and the similarity factor $\mathrm{F} 2$ and the difference factor $\mathrm{F} 1$ are calculated. Based on all the three dissolutions, the graph is constructed retrieving the data from the database. From the graph above, we can conclude that the drug reaction of all the three dissolutions i.e. at the fast, the medium and the intermediate converges is more or less the same. When compared with Math work this proves easier for the user and though loads of PKPD tools are available in the market, they remain unused owing to the complexity. The attempt made in this chapter clearly draws out that any end user feels contented to avail this tool.

\section{CONCLUSION AND FUTURE WORK}

The high cost to bring drugs to the market has been mainly due to the large investment required during the "clinical path". This declined the new drug submissions for regulatory approvals. Another main reason that propped up was the clinical fails of drugs and the inability to predict a drug's response in advance in the development process. The US Food and Drug Administration (FDA) in 2004 released a white paper that addressed this issue and proposed the employment of valid biomarkers, the system approach and the novel trial design to improve the drug development and decision-making process.

A good number of human infection units are currently treatable by medicine. The Clinical trials keep within the gold customary, empirical approach guiding drug preparation policy and the smart issues like dosing regimens. In silico simulations the supported of the drug treatment outcome has the potential to play a major adjuvant role in coming up with and guiding these preparation practices. The tool is hoped to pave way to the welfare of patients and significantly reduce the cost of drugs. This will definitely rule out the complex issues in making the new drug to emerge. The softwares VB and SQL8 is more user friendly and it will satisfy all the customer requirements. This $\mathrm{PK} / \mathrm{PD}$ kit provides an extensible framework to conduct numerous studies on drug dosage. By combining all biological data into a single platform, it provides good effect of foreign chemicals on human body. This PK/PD kit will definitely be a significant tool for testing of drugs in human health and safety against environmental chemical body risks.

\section{REFERENCES}

[1] Dong, Jennifer Q, Chen, Bin, Gibbs Megan A, Emery, Maurice, John P., Applications of Computer-Aided Pharmacokinetic and Pharmacodynamic Methods from Drug Discovery Through Registration, Volume 4, Number 1, pp. 54-66(13), Bentham Science Publishers (2008).

[2] Reisfeld B, Mayeno A, Lyons M, Yang R: Physiologically-based pharmacokinetic and pharmacodynamics modeling. In Computational Toxicology: Risk Assessment for Pharmaceutical and Environmental Chemicals. Edited by Ekins S, Wiley, pp 33, ( 2007).

[3] Lakshmi Kamath, Modeling Success in PK/PD Testing, 39(10):1954-60 (2011).

[4] Lake AD, Novak P, Fisher CD, Jackson JP, Hardwick RN, Billheimer, Klimecki, Cherrington NJ, Analysis of global absorption, distribution, metabolism, and elimination gene expression in the progressive stages of human nonalcoholic fatty liver disease, pp. 464-469 (2011).

[5] Phillip J. Bergen, Jian Li and Roger L, Dosing of colistin, Department of Pharmacology and Toxicology, Tucson, AZ 85721, USA (2011)

[6] Czock D, Keller F, Mechanism-based pharmacokineticpharmacodynamics modeling of antimicrobial drug effects. J Pharmacokinetic Pharmacodynamic 34: pp. 727-751 (2007).

[7] Leon Aarons, Software for Population Pharmacokinetics and Pharmacodynamics, Volume 36, Issue 4, pp. 255-264 (1999).

[8] Robert J. Bauer, Serge Guzy, Chee Ng, A survey of population analysis methods and software for complex pharmacokinetic and pharmacodynamics models with examples, The AAPS Journal , Volume 9, Issue 1, pp. E60E83 (2007).

[9] Beal SL, Sheiner LB, Methodology of population pharmacokinetics, Drug fate and metabolism: methods and techniques. New York: Marcel Dekker, 135-83. (1985).

[10] Racine-Poon A, Smith AFM. Population models, Statistical methodology in the pharmaceutical sciences. New York: Marcel Dekker, 139-62 (1990).

[11] Mallet A. A maximum likelihood estimation method for random coefficient regression models. Biometrika 73: 64556. CrossRef (1986).

[12] Lindstrom MT, Bates DM. Nonlinear mixed effects models for repeated measures data. Biometrics; 46: 67387. CrossRef (1990)

[13] Beal SL, Sheiner LB. NONMEM Users Guides (I-VIII) San Francisco: University of California NONMEM Project Group (1998).

[14] Yong Zhang, MeirongHuo, Jianping Zhou, ShaofeiXie, PKSolver: An add-in program for pharmacokinetic and pharmacodynamic data analysis in Microsoft Excel, Computer Models and Programs in Biomedicine, Volume 99, Issue 3, pp. 306-314 (2010)

[15] Iris Rajman, PK/PD modeling and simulations: utility in drug development, Novartis Pharma AG, WSJ-210.6.29, CH4056 Basel, Switzerland (2012).

[16] J.Y. Chien, Stuart Friedrich, Michael A, Heathman, Dinesh P. de Alwis, and Vikram Sinha Pharmacokinetics/pharmacodynamics and the stages of drug development: Role of modeling and simulation, AAPS J. September; 7(3): E544-E559 (2005).

[17] SimBiology model for PK/PD of anticancer drugs by Asawari Samant, Matlab Central (2011).

[18] Tan YM, Liao KH, Clewell r H J: Reverse dosimetry: interpreting trihalomethanes biomonitoring datausing physiologically based pharmacokinetic modeling. J Expo Sci Environ Epidemiol pp. 591-603, (2007). 
[19] Marino DJ, Clewell HJ, Gentry PR, Covington TR, Hack CE, David RM, Morgott DA: Revised assessment of cancer risk to dichloromethane: Part I Bayesian PBPK and doseresponse modeling in mice. Regul Toxicol Pharmacol, pp.273-300, (2006)

[20] Lyons MA, Yang RSH, Mayeno AN, Reisfeld B: Computational toxicology of chloroform: reverse dosimetry using Bayesian inference, Markov chain Monte Carlo simulation, and human biomon-itoring data. Environmental Health Perspectives,pp. 116-124, (2008).

[21] Gelman A, Bois FY, Jiang J: Physiological pharmacokinetic analysis using population modeling and informative prior distributions. Journal of American Statistical Association, pp. 1400-1412, (1996).

[22] Tan C, Liao K, Clewell H: Physiologically Based Pharmacokinetic Modeling as a Tool to Interpret Human Biomonitoring Data. CIIT Activities, (2005).

[23] Liao KH, Tan YM, Clewell r H J: Development of a screening approach to interpret human biomonitor-ing data on volatile organic compounds: reverse dosimetry on biomonitoring data for trichloroethy-lene. Risk Anal, pp. 2736 , (2007).

[24] Selly, J.P.,G.L. Amidon, W.H. Barr, L.Z. Benet, J.E. Carter, J.R. Robinson, V.P. Shah and A.Yacobi, "in Vitro and In Vivo Testing and Correlation for Oral Controlled/Modified Release Dosage Forms", Pharmaceutical Research, 7:975-982 (1990). 


\section{APPENDIX}

\subsection{Tables}

Table 1 Dissolution Profile of Drug $\mathbf{X}$ for Fast, Intermediate and Slow

\begin{tabular}{|l|l|l|l|l|l|l|l|}
\hline \multicolumn{9}{|c|}{ Dissolution profile of Drug X for fast medium } \\
\hline $\begin{array}{l}\text { Time( } \\
\text { min) }\end{array}$ & $\begin{array}{l}\text { Dissolution value of th ref } \\
\text { batch at time t (Ref) }\end{array}$ & $\begin{array}{l}\text { Dissolution value of the test } \\
\text { batch at time t(Test) }\end{array}$ & $\begin{array}{l}\text { (Ref- } \\
\text { test) }\end{array}$ & (Ref-test) 2 & T mid & $\Delta$ M & $T^{*} \Delta M$ \\
\hline 0 & 0 & 0 & 0 & 0 & 0 & 0 & 0 \\
\hline 0.5 & 41 & 38 & 3 & 9 & 0.25 & 41 & 10.25 \\
\hline 1 & 47.08 & 43 & 4.08 & 16.6464 & 0.75 & 6.08 & 4.56 \\
\hline 2 & 60 & 54 & 6 & 36 & 1.5 & 12.92 & 19.38 \\
\hline 3 & 65 & 64 & 1 & 1 & 2.5 & 5 & 12.5 \\
\hline 4 & 78.02 & 75 & 3.02 & 9.1204 & 3.5 & 13.02 & 45.57 \\
\hline 5 & 89.34 & 84 & 5.34 & 28.5156 & 4.5 & 11.32 & 50.94 \\
\hline 6 & 93.98 & 94 & -0.02 & 0.0004 & 5.5 & 4.64 & 25.52 \\
\hline 7 & 99.05 & 97 & 2.05 & 4.2025 & 6.5 & 5.07 & 32.955 \\
\hline
\end{tabular}

\begin{tabular}{|l|l|l|l|l|l|l|l|}
\hline \multicolumn{7}{|c|}{ Dissolution profile of Drug X for intermediate medium } \\
\hline $\begin{array}{l}\text { Time( } \\
\text { min) }\end{array}$ & $\begin{array}{l}\text { Dissolution value of } \\
\text { th ref batch at time t } \\
\text { (Ref) }\end{array}$ & $\begin{array}{l}\text { Dissolution value of } \\
\text { the test batch at time } \\
\text { (Test) }\end{array}$ & $\begin{array}{l}\text { (Ref- } \\
\text { test) }\end{array}$ & $\begin{array}{l}\text { (Ref- } \\
\text { test) } 2\end{array}$ & $\begin{array}{l}\text { T } \\
\text { mid }\end{array}$ & $\Delta \mathrm{M}$ & $T^{*} \Delta \mathrm{M}$ \\
\hline 0 & 0 & 0 & 0 & 0 & 0 & 0 & 0 \\
\hline 0.5 & 38 & 37 & 1 & 1 & 0.25 & 38 & 9.5 \\
\hline 1 & 45 & 42 & 3 & 9 & 0.75 & 7 & 5.25 \\
\hline 2 & 59 & 53 & 6 & 36 & 1.5 & 14 & 21 \\
\hline 3 & 64 & 62 & 2 & 4 & 2.5 & 5 & 12.5 \\
\hline 4 & 79 & 74 & 5 & 25 & 3.5 & 15 & 52.5 \\
\hline 5 & 87 & 86 & 1 & 1 & 4.5 & 8 & 36 \\
\hline 6 & 94 & 92 & 2 & 4 & 5.5 & 7 & 38.5 \\
\hline 7 & 99.02 & 98 & 1.02 & 1.0404 & 6.5 & 5.02 & 32.63 \\
\hline $\begin{array}{l}\text { Similarity factor f2 } \\
\text { Difference factor f1 }\end{array}$ & & 21.02 & 81.0404 & & 99.02 & 207.88 \\
\hline
\end{tabular}




\begin{tabular}{|c|c|c|c|c|c|c|c|}
\hline \multicolumn{8}{|c|}{ Dissolution profile of Drug $\mathrm{X}$ for slow medium } \\
\hline Time(min) & $\begin{array}{l}\text { Dissolution value of } \\
\text { th ref batch at time } t \\
\text { (Ref) }\end{array}$ & $\begin{array}{l}\text { Dissolution value of } \\
\text { the test batch at time } \\
t(\text { Test) }\end{array}$ & (Ref-test) & $($ Ref-test)^2 & T mid & $\Delta \mathrm{M}$ & $\mathrm{T}^{*} \Delta \mathrm{M}$ \\
\hline 0 & 0 & 0 & 0 & 0 & 0 & 0 & 0 \\
\hline 0.5 & 41 & 40 & 1 & 1 & 0.25 & 41 & 10.25 \\
\hline 1 & 43 & 45 & -2 & 4 & 0.75 & 2 & 1.5 \\
\hline 2 & 61 & 57 & 4 & 16 & 1.5 & 18 & 27 \\
\hline 3 & 64 & 63 & 1 & 1 & 2.5 & 3 & 7.5 \\
\hline 4 & 78 & 76 & 2 & 4 & 3.5 & 14 & 49 \\
\hline 5 & 88 & 88 & 0 & 0 & 4.5 & 10 & 45 \\
\hline 6 & 93.98 & 92 & 1.98 & 3.9204 & 5.5 & 5.98 & 32.89 \\
\hline \multirow[t]{2}{*}{7} & 99.05 & 98 & 1.05 & 1.1025 & 6.5 & 5.07 & 32.955 \\
\hline & 568.03 & & 9.03 & 31.0229 & & 99.05 & 206.095 \\
\hline \multicolumn{7}{|c|}{ Similarity factor F2 } & $\begin{array}{l}83.79834889 \\
1.589704769\end{array}$ \\
\hline
\end{tabular}

\subsection{Figures}

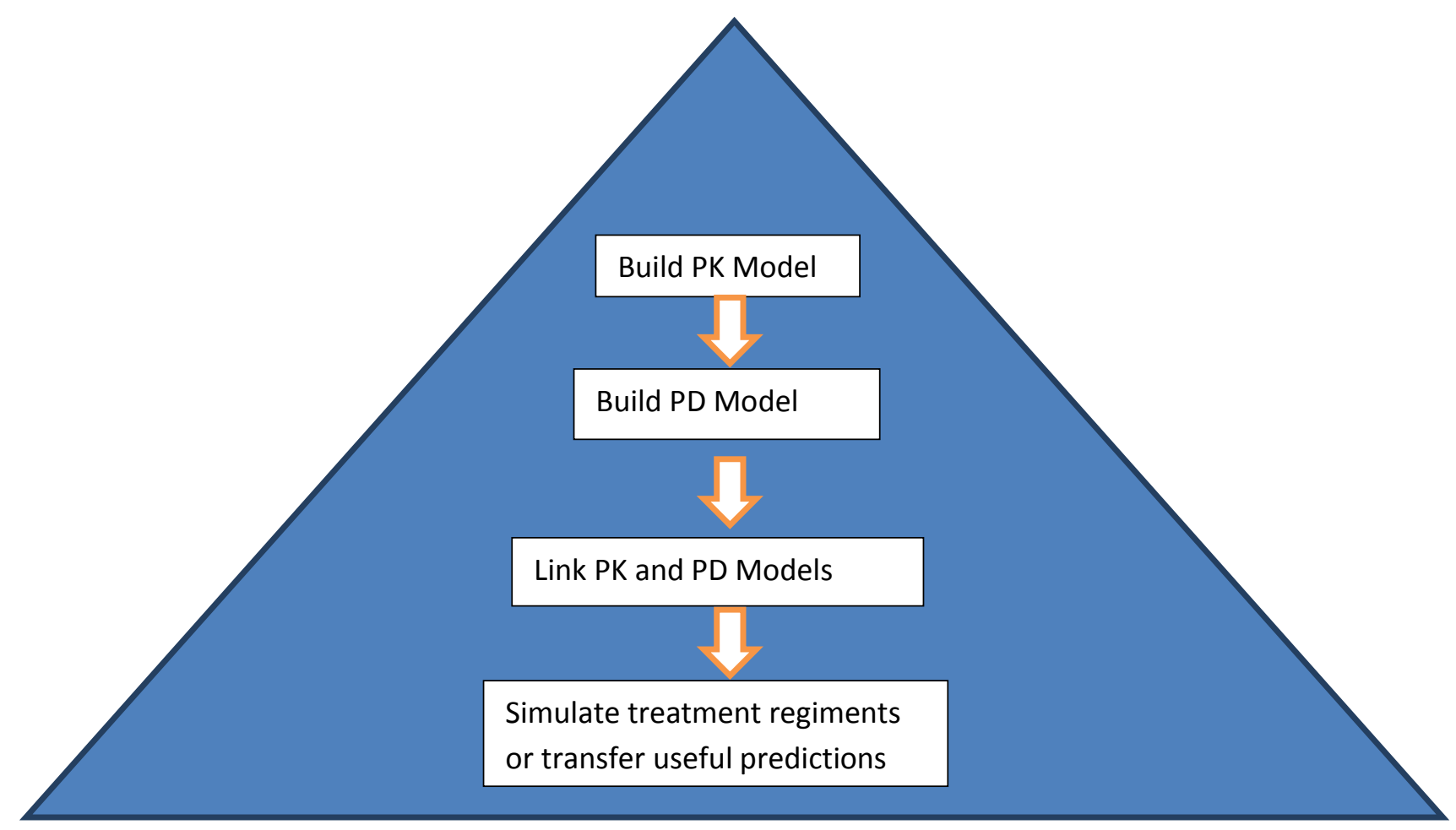

Fig 1. STREAMLINING THE PROCESS 

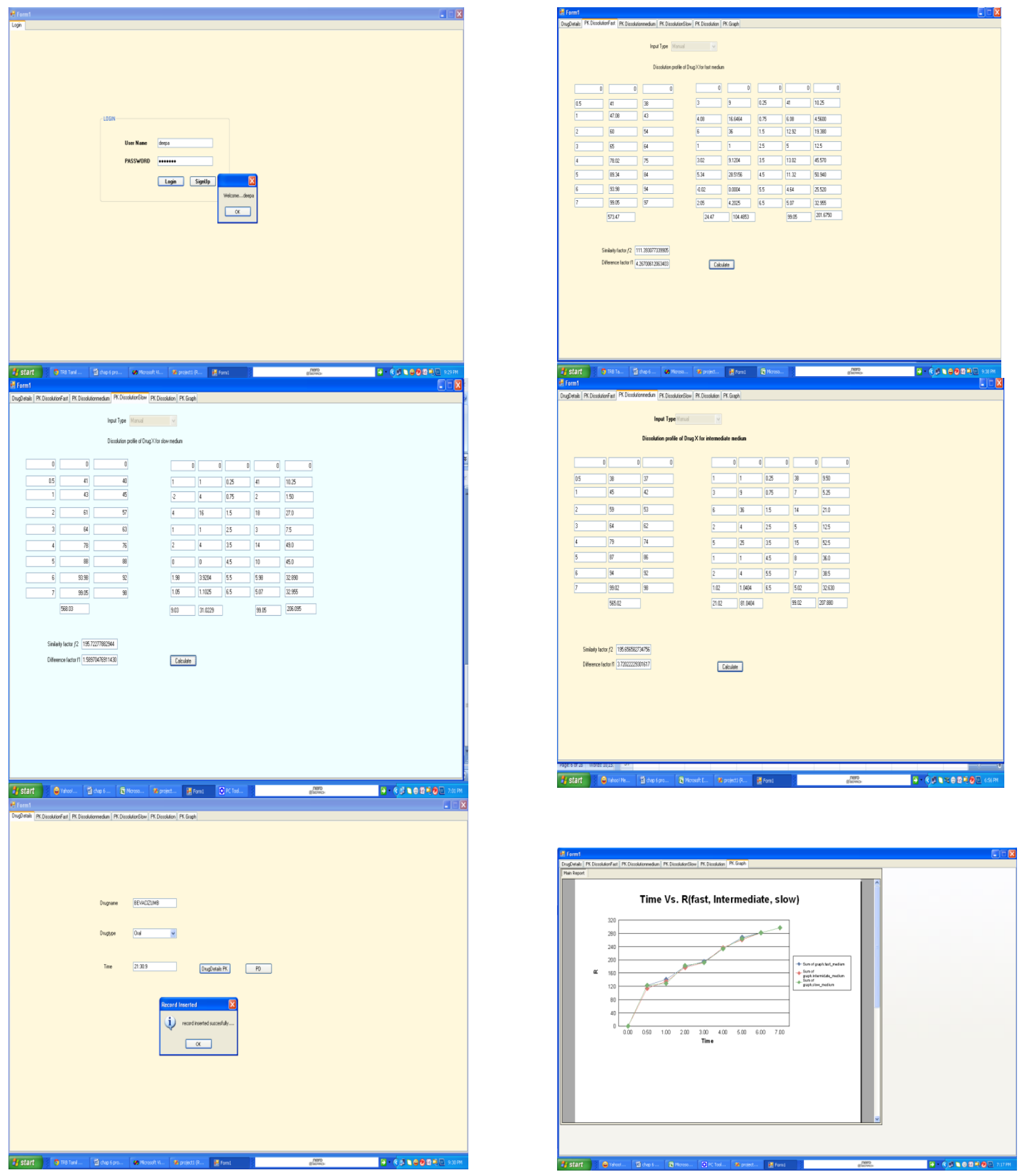

Fig 2: Snapshots showing the working of tool 